\title{
COOLING OF NEUTRON STARS WITH COLOR SUPERCONDUCTING QUARK CORES *
}

\author{
D. BLASCHKE \\ Fachbereich Physik, Universität Rostock, D-18051 Rostock, Germany \\ Bogoliubov Laboratory for Theoretical Physics, JINR Dubna, 141980 Dubna, \\ Russia \\ email: david@thsun1.jinr.ru \\ D. N. VOSKRESENSKY \\ Theory Division, GSI mbH, D-64291 Darmstadt, Germany \\ Moscow Institute for Physics and Engineering, 115409 Moscow, Russia \\ H. GRIGORIAN \\ FB Physik, Universität Rostock, D-18051 Rostock, Germany \\ Department of Physics, Yerevan State University, Alex Manoogian Str. 1, \\ 375025 Yerevan, Armenia
}

\begin{abstract}
We show that within a recently developed nonlocal, chiral quark model the critical densities for a phase transition to color superconducting quark matter under neutron star conditions can be low enough that these phases occur in compact star configurations with masses below $1.4 M_{\odot}$. We study the cooling of these objects in isolation for different values of the gravitational mass and thus different composition and structure of the interior. Our equation of state allows for a $2 \mathrm{SC}$ phase with a large quark gap $\Delta \sim 100 \mathrm{MeV}$ for $u$ and $d$ quarks of two colors, a normal quark matter phase and their coexistence in a mixed phase within the hybrid star interior. We argue that, if the phases with unpaired quarks were allowed, the corresponding hybrid stars would cool too fast to describe the neutron star cooling data existing by today. We incorporate other attractive channels permitting a weak pairing of the residual quarks which remained unpaired in the 2SC phase and demonstrate that the model does not contradict the cooling data if the weak pairing gaps are of the order of $0.1 \mathrm{MeV}$.
\end{abstract} *Conference Proceedings of the KIAS-APCTP International Symposium in Astro-
Hadron Physics Compact Stars: Quest for New States of Dense Matter 


\section{Introduction}

In the recent paper ${ }^{1}$, hereafter $\mathrm{BGV}$, we have reinvestigated the cooling of neutron stars (NS) within a purely hadron model, i.e., ignoring the possibility of quark cores in NS interiors. We have demonstrated that the NS cooling data available by today can be well explained within the "Nuclear medium cooling scenario", i.e., if one includes medium effects in the emissivity and takes into account a suppression of the $3 P_{2}$ neutron gap. In a subsequent work ${ }^{2}$ we have shown that this result does not exclude the possibility that neutron stars might possess large quark matter cores that extend up to more than half of the star radius. Such a hybrid structure gives room for a whole variety of additional scenarios of compact star cooling which fall into two classes: either nuclear and quark matter phases have similar cooling behavior (homgeneous cooling) or the faster cooling of the one phase is compensated by the slower cooling of the other (inhomogeneous cooling). In the present contribution we will report on our results within the former, homgeneous cooling scenario of hybrid stars and what implications the comparison with present-day cooling data may provide for the EoS and transport properties of quark matter.

\section{Color superconductivity}

The quark-quark interaction in the color anti-triplet channel is attractive driving the pairing with a large zero-temperature pairing gap $\Delta \sim 100 \mathrm{MeV}$ for the quark chemical potential $\mu_{q} \sim(300 \div 500) \mathrm{MeV}$, cf. ${ }^{3,4}$, see review ${ }^{5}$ and Refs therein. The attraction comes either from the one-gluon exchange, or from a non-perturbative 4-point interaction motivated by instantons ${ }^{6}$ or from non-perturbative gluon propagators ${ }^{7}$. Various phases are possible. The so called 2-flavor color superconductivity (2SC) phase allows for unpaired quarks of one color, say blue. There may also exist a color-flavor locked (CFL) phase ${ }^{8}$ for not too large values of the dymanical strange quark mass or other words for very large values of the baryon chemical potential ${ }^{9}$, where the color superconductivity (CSC) is complete in the sense that the diquark condensation produces a gap for quarks of all three colors and flavors. The value of the gap is of the same order of magnitude as that in the two-flavor case. There exist other attractive quark pairing channels for quarks that can't participate in 2SC and CFL pairing. These weak pairing channels are characterized by gaps typically in the interval $\sim 10 \mathrm{keV} \div 1 \mathrm{MeV}$, and a prominent example which will be used in the present work is the spin-1 pairing channel of single color diquarks in the 
isospin singlet state, also named as color-spin locking (CSL) phase.

The high-density phases of QCD at low temperatures may exist in the interiors of hybrid stars affecting their cooling, rotation and magnetic field evolution, cf. ${ }^{11,12,13,14,15}$.

\section{Hybrid stars}

In describing the hadronic part of the hybrid star we exploit the Argonne $V 18+\delta v+U I X^{*}$ model of the EoS given in ${ }^{16}$, which is based on the most recent models for the nucleon-nucleon interaction with the inclusion of a parameterized three-body force and relativistic boost corrections. Actually we adopt here an analytic parameterization of this model by Heiselberg and Hjorth-Jensen ${ }^{17}$, hereafter HHJ. The latter uses a compressional part with the incompressibility $K \simeq 240 \mathrm{MeV}$, and a symmetry energy fitted to the data around nuclear saturation density that smoothly incorporates causality at high densities. The density dependence of the symmetry energy is very important since it determines the value of the threshold density for the DU process $\left(n_{c}^{\mathrm{DU}}\right)$. The HHJ EoS fits the symmetry energy to the original Argonne $V 18+\delta v+U I X^{*}$ model yielding $n_{c}^{\mathrm{DU}} \simeq 5.19 n_{0}$ $\left(M_{c}^{\mathrm{DU}} \simeq 1.839 M_{\odot}\right)$.

The 2SC phase occurs at lower baryon densities than the CFL phase, see ${ }^{18,19}$. For applications to compact stars the omission of the strange quark flavor is justified by the fact that central chemical potentials in star configurations do barely reach the threshold value at which the mass gap for strange quarks breaks down and they appear in the system ${ }^{22}$.

We will focus on the model of the quark EoS developed in ${ }^{23}$. The Gaussian, Lorentzian and NJL type cutoff formfactors were studied. The Lorentzian interpolates between a soft (Gaussian type, $\alpha \sim 2$ ), and a hard (NJL, $\alpha>30$ ) depending on the value of the parameter $\alpha$. We will further work out two possibilities of the Gaussian and the Lorentzian formfactors.

In some density interval at the first order phase transition there may appear the region of the mixed phase, see ${ }^{21}$. Ref. ${ }^{21}$ disregarded finite size effects, such as surface tension and charge screening. Refs ${ }^{24}$ on the example of the hadron-quark mixed phase have demonstrated that finite size effects might play a crucial role substantially narrowing the region of the mixed phase or even forbidding its appearance. Therefore we omit the possibility of the hadron-quark mixed phase in our model where the quark phase arises by the Maxwell construction. For the case of the Gaussian formfactor the quark core appears for $n>n_{c}=0.34 \mathrm{fm}^{-3}\left(M>0.852 M_{\odot}\right)$, 


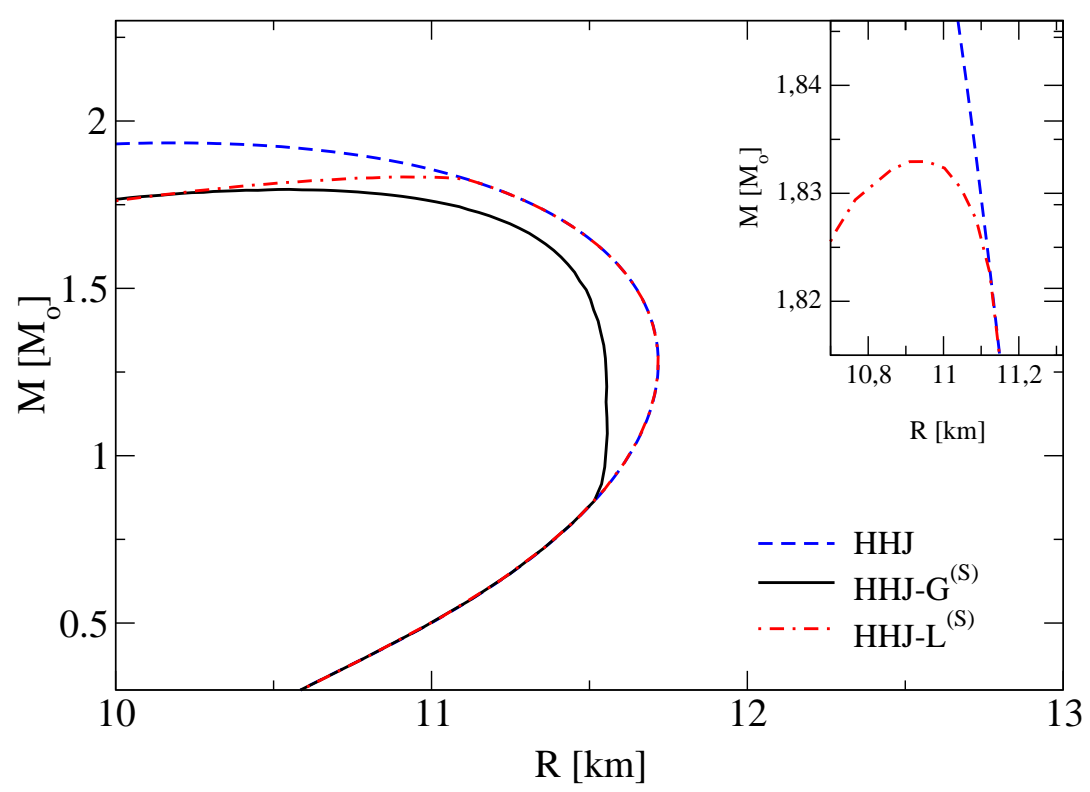

Figure 1. Mass - radius relations for compact star configurations with different EoS: purely hadronic star with HHJ EoS (dashed line), stable hybrid stars with HHJ - SM $\mathrm{G}_{\text {(S) }}$ EoS (solid line) and with HHJ - $\mathrm{SM}_{\mathrm{L}}^{(\mathrm{S})}$ EoS (dash-dotted line).

for the Lorentzian formfactor $n>n_{c}=0.80 \mathrm{fm}^{-3}\left(M>1.81 M_{\odot}\right)$. In the following, we will not further discuss the Lorentzian case which gives a marginal quark core in a small mass range only, see Fig. 1.

A large difference between chemical potentials of $u$ and $d$ quarks forbids the pure 2SC phase, cf. $^{23}$. The CFL phase is still not permitted at such densities. Ignoring the possibility of a weak coupling we have two possibilities: either the quark matter for $n>n_{c}$ is in the normal phase, or there appears a region of the $2 \mathrm{SC}$ - normal quark mixed phase. Following ${ }^{21}$, Ref. ${ }^{22}$ considered the latter possibility disregarding finite size effects and has found the possibility of a wide region (for Gaussian formfactor) of the 2SC - normal quark mixed phase instead of a pure 2SC phase. In the given case arguments of ${ }^{24}$ are relaxed since the surface tension on the $2 \mathrm{SC}$ - normal quark boundary should be much less compared to that for the quark - hadron boundary. Indeed, the surface tension is proportional to 
the difference of the energies of two phases, being very small in the given case, $\propto\left(\Delta / \mu_{q}\right)^{2} \ll 1$.

In Fig. 1 we present the mass-radius relation for hybrid stars with HHJ vs. SM EoS. Two sets of configurations given by Gaussian (solid lines) and Lorentzian (dash-dotted lines) formfactors are stable, see also Ref. ${ }^{23}$, where similar results for the nonlinear Walecka model EoS (RMF) have been found.

If one switches on the possibility of the weak coupling, e.g. the CSL pairing channel, see ${ }^{10,19,20}$ all the quarks in the normal phase may acquire a corresponding spin-1 pairing gap, typically $\Delta \sim 10 \mathrm{keV} \div 10 \mathrm{MeV}$. In such a way all the quarks may get paired, either strongly in the $2 \mathrm{SC}$ channel or weakly in the CSL one.

\section{Cooling}

For the calculation of the cooling of the hadron part of the hybrid star we adopt the same model as in BGV. The main processes are medium modified Urca (MMU) and pair breaking and formation (PBF) processes. The HHJ EoS was adopted. In Fig. 2 (Fig. 20 of BGV) we show the cooling of different NS calculated within the hadron model of BGV. We use a fit-law for the relation between surface and interior temperatures, see BGV. Possibilities of the pion condensation and of the other so called exotic processes are for simplicity suppressed. Direct Urca is irrelevant in this model up to very large NS mass $M>1.839 M_{\odot} .1 S_{0}$ neutron and proton gaps are taken the same as in paper ${ }^{25}$, whereas $3 P_{2}$ neutron gap is suppressed by the factor 0.1 , see Fig. 4 of BGV.

For the calculation of the cooling of the quark part of the hybrid star we are basing on the model ${ }^{13}$. We include the most efficient processes: the quark direct Urca (QDU) processes on unpaired quarks, the quark modified Urca (QMU), the quark bremsstrahlung (QB), the electron bremsstrahlung (EB) Following ${ }^{26}$ we include the emissivity of the quark pair formation and breaking (QPFB) processes. The specific heat incorporates the quark contribution, the electron contribution and the gluon-photon contribution. In the CSL phase ${ }^{20}$ the specific heat is proportional to $T^{2}$. This new term does not significantly affect the total specific heat since the main contribution comes from electrons. The heat conductivity contains quark, electron and gluon terms.

We are basing on the picture presented in Fig. 2 and add the contribution of the quark core. For the Lorentzian formfactor the quark core 


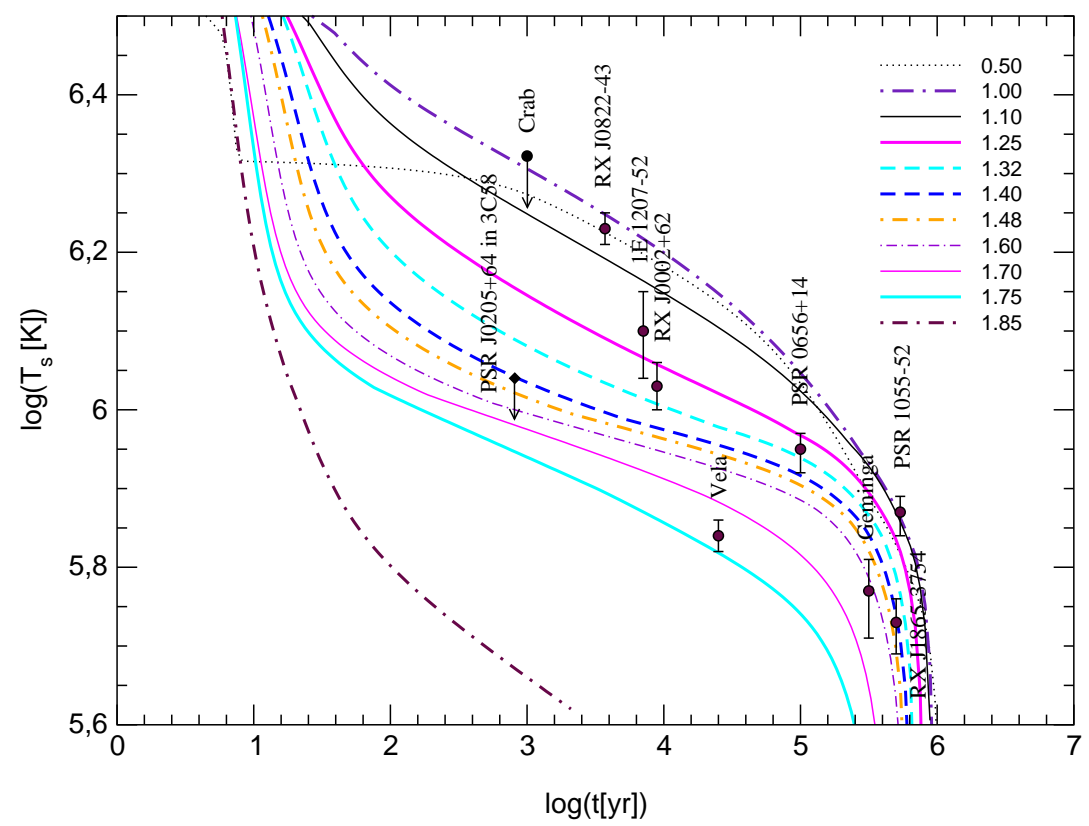

Figure 2. Cooling curves according to the nuclear medium cooling scenario, see Fig. 20 of BGV. The labels correspond to the gravitational masses of the configurations in units of the solar mass.

appears only for $M>1.81 M_{\odot}$, see Fig. 1 . The existing cooling data are not affected, thereby.

For the Gaussian formfactor the quark core occurs already for $M>$ $0.852 M_{\odot}$ according to the model ${ }^{23}$, see Fig. 1 . Most of the relevant NS configurations (see Fig. 2) are then affected by the presence of the quark core. First we check the possibility of the $2 \mathrm{SC}+$ normal quark phases.

Fig. 3 shows the cooling curves calculated with the Gaussian ansatz. The variation of zero temperature gaps for the strong pairing of quarks within 2 SC phase in the interval $\Delta \sim 20 \div 200 \mathrm{MeV}$ only slightly affects the results. The main cooling process is the QDU process on normal quarks. We see that the presence of normal quarks leads to too fast cooling. The data could be explained only if all the masses lie in a very narrow interval $\left(0.82<M / M_{\odot}<0.90\right.$ in our case $)$. The existence of only a very narrow 


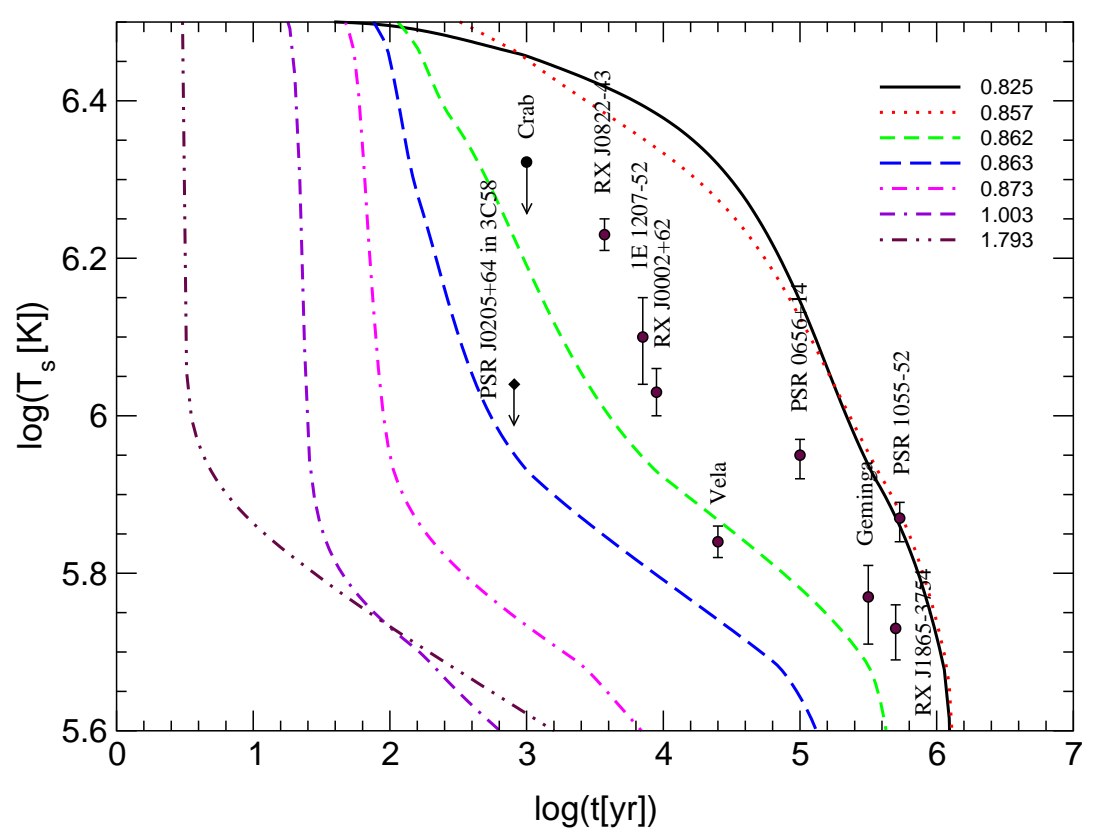

Figure 3. Cooling curves for hybrid star configurations with Gaussian quark matter core in the 2SC phase. The labels correspond to the gravitational masses of the configurations in units of the solar mass.

mass interval in which the data can be fitted seems us unrealistic as by itself as from the point of view of the observation of the NS with different masses: $M \simeq 1.41 M_{\odot}$ and $M \simeq 1.25 M_{\odot}$, cf. ${ }^{27}$. Thus the data can't be satisfactorily explained.

In Fig. 4 we permit the weak pairing for all quarks which were assumed to be unpaired. We use $\Delta \simeq 1 \mathrm{MeV}$ for the corresponding quark gap. Fig. 4 demonstrates too slow cooling.

In Fig. 5 we again allow for the weak pairing of those quarks which were assumed to be unpaired in Fig. 3, but now we use $\Delta=50 \mathrm{keV}$ for the corresponding CSL quark gap. The data are appropriately fitted. The "slow cooling" data are explained by the cooling of NS either without quark core $\left(M \sim 0.5 M_{\odot}\right.$, see Fig. 2$)$ or with a quark core. Although the majority of experimental cooling points are covered by NS masses in a very narrow 


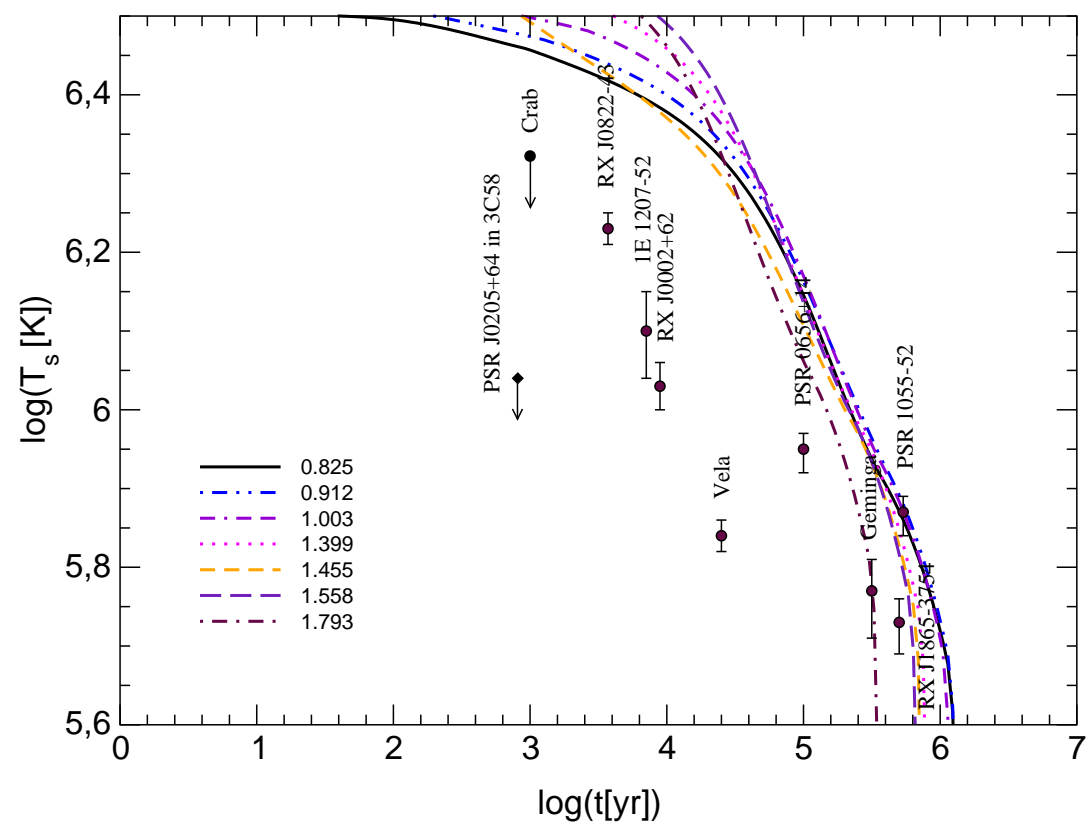

Figure 4. Cooling curves for hybrid star configurations with Gaussian quark matter core in the 2SC+CSL phase. The weak pairing gap is $1 \mathrm{MeV}$. The labels correspond to the gravitational masses of the configurations in units of the solar mass.

mass interval $M=0.86 \div 0.87 M_{\odot}$ the difference with Fig. 3 is crucial. We see that with the higher masses, up to $1.6 M_{\odot}$ one may cover the rapid cooling point (Vela). Besides, the variation of parameters allows to shift all the curves up what permits to essentially broaden the mass interval that would cover the data. E.g. with the so called Tsuruta law for the $T_{s}-T_{i n}$ relation the corresponding mass interval is $M=0.86 \div 1.79 M_{\odot}$. With these remarks we showed that the $2 \mathrm{SC}+\mathrm{CSL}+$ hadron scenario allows to fit the data as well as the purely hadronic scenario, see ${ }^{1}$. To be more realistic one should further inlcude density dependences of the gaps what we intend to do in the forthcoming publication. 


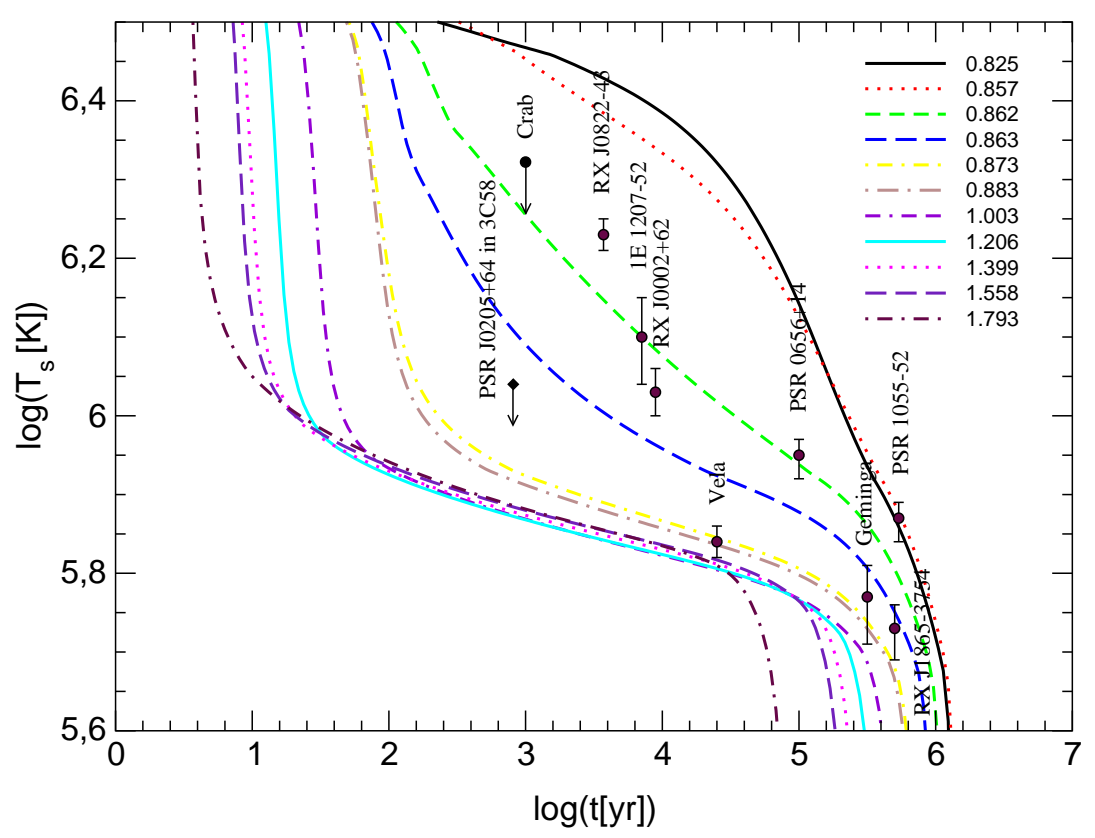

Figure 5. Same as Fig. 4 but with a weak pairing gap of $50 \mathrm{keV}$.

\section{Conclusion}

Concluding, we demonstrated that the present day cooling data can be explained not only by a purely hadronic structure of NS interiors but also by a hybrid one with a complex pairing pattern, where quarks are partly strongly paired within the $2 \mathrm{SC}$ channel, and partly weakly paired within the CSL channel with gaps $\Delta \lesssim 50 \mathrm{keV}$. We conclude also that our choice of a density-independent weak pairing gap could be the reason why the mass interval for explaining slow and intermediate cooling data is very narrow. As it is well-known that the CSL gap should have a strong density dependence ${ }^{20}$, the fastening of the cooling by increasing the star masses should be partly compensated for by the corresponding increase of the weak pairing gap. Corresponding calculations are under way. 


\section{Acknowledgement}

H.G. and D.V. acknowledge the hospitality and support of Rostock University. The work of H.G. has been supported in part by the Virtual Institute of the Helmholtz Association under grant No. VH-VI-041, that of D.V. has been supported in part by DFG grant No. 436 RUS 17/117/03 and by RFBR grant NNIO-03-02-04008. D.B. thanks the organizers of the workshop, in particular D.-K. Hong and C.-H. Lee, for their invitation and support.

\section{References}

1. D. Blaschke, H. Grigorian, and D.N. Voskresensky, Cooling of Neutron Stars. Hadronic Model, arXiv:astro-ph/0403170

2. D. Blaschke, H. Grigorian, and D.N. Voskresensky, Cooling of Neutron Stars. Hybrid Model with 2SC and CSL Quark Matter Phases, Preprint MPG-VTUR 247/04 (2004).

3. M. Alford, K. Rajagopal, and F. Wilczek, Phys. Lett. B 422, 247 (1998).

4. R. Rapp, T. Schäfer, E.V. Shuryak, and M. Velkovsky, Phys. Rev. Lett. 81, 53 (1998).

5. K. Rajagopal, and F. Wilczek, "The Condensed Matter Physics of QCD", in: B.L. Ioffe Festschrift At the Frontier of article Physics / Handbook of QCD, vol. 3, edited by M. Shifman, World Scientific, Singapore, 2001, p. 2061

6. D. Diakonov, H. Forkel, and M. Lutz, Phys. Lett. B 373, 147 (1996); G.W. Carter, and D. Diakonov, Phys. Rev. D 60, 016004 (1999); R. Rapp, E. Shuryak, and I. Zahed, Phys. Rev. D 63, 034008 (2001).

7. D. Blaschke, and C.D. Roberts, Nucl. Phys. A 642, 197 (1998); J.C.R. Bloch, C.D. Roberts, and S.M. Schmidt, Phys. Rev. C 60, 65208 (1999).

8. M. Alford, K. Rajagopal, and F. Wilczek, Nucl. Phys. B 357, 443 (1999); T. Schäfer, and F. Wilczek, Phys. Rev. Lett. 82, 3956 (1999).

9. M. Alford, J. Berges, and K. Rajagopal, Nucl. Phys. B 558, 219 (1999).

10. M. Alford, J. Bowers, J. Cheyne, and G.A. Cowan, Phys. Rev. D 67, 054018 (2003).

11. D. Blaschke, T. Klähn, and D.N. Voskresensky, Astrophys. J. 533, 406 (2000).

12. D. Page, M. Prakash, J.M. Lattimer and A. Steiner, Phys. Rev. Lett. 85 2048 (2000).

13. D. Blaschke, H. Grigorian, and D.N. Voskresensky, Astron. \& Astrophys. 368, 561 (2001).

14. D. Blaschke, D. M. Sedrakian, and K. M. Shahabasian, Astron. \& Astrophys. 350, L47 (1999); D.M. Sedrakian, D. Blaschke, K.M. Shahabasyan, and D.N. Voskresensky, Astrofizika 44, 443 (2001).

15. K. Iida, and G. Baym, Phys. Rev. D 63, 074018 (2001); Phys. Rev. D 65, 014022 (2002); Phys. Rev. D 66, 014015 (2002).

16. A. Akmal, V.R. Pandharipande, and D.G. Ravenhall, Phys. Rev. C 58, 1804 
(1998).

17. H. Heiselberg, and M. Hjorth-Jensen, Astrophys. J. 525, L45 (1999).

18. A.W. Steiner, S. Reddy, and M. Prakash, Phys. Rev. D 66, 094007 (2002).

19. F. Neumann, M. Buballa, and M. Oertel, Nucl. Phys. A714, 481 (2003).

20. M. Buballa, J. Hosek and M. Oertel, Phys. Rev. Lett. 90 (2003) 182002

21. N.K. Glendenning, Phys. Rev. D 461274 (1992); N.K. Glendenning, Phys. Rep. 342, 393 (2001).

22. C. Gocke, D. Blaschke, A. Khalatyan, and H. Grigorian, arXiv:hep$\mathrm{ph} / 0104183$.

23. D. Blaschke, S. Fredriksson, H. Grigorian, and A. Öztas, Nucl. Phys. A (2004), in press; [arXiv:nucl-th/0301002]; H. Grigorian, D. Blaschke, and D.N. Aguilera, [arXiv:astro-ph/0303518].

24. D.N. Voskresensky, M. Yasuhira, and T. Tatsumi, Phys. Lett. B 541, 93 (2002); Nucl. Phys. A 723, 291 (2002).

25. T. Takatsuka, and R. Tamagaki, arXiv:nucl-th/0402011.

26. P. Jaikumar, and M. Prakash, Phys. Lett. B 516, 345 (2001).

27. A.G. Lyne, et al., arXiv:astro-ph/0401086. 